\title{
Effects of enriching goat meat with $n-3$ polyunsaturated fatty acids on meat quality and stability
}

\begin{abstract}
The effect of enriching goat meat with $\mathrm{n}$ i 3 polyunsaturated fatty acids (PUFA) on meat quality, sensory evaluation, and lipid oxidative stability at different postmortem aging times was investigated. Samples from longissimus thoracis muscle of Boer bucks $(n=24)$ fed diets containing $0 \%$ (control, L0), 10\% (L10), or 20\% (L20) whole linseed were collected for analysis. For meat quality and oxidative stability, sub samples were subjected to 1-day or 7day postmortem aging period. Feeding linseed had successfully increased n $\bar{\imath} 3$ PUFA in goat meat. The increments in 18:3 n i 3 were $682 \%$ and $975 \%$ for L10 and L20, respectively, compared to L0. The increments in total n $\bar{\imath} 3$ PUFA were 444\% and 590\%for L10 and L20, respectively, compared with $\mathrm{L} 0$. There was no effect on cooking loss, shear force and color at 1-day aging time. However, at 7-day of aging time the color was affected. Subjectively, the tenderness, juiciness and overall acceptability of L20 meat were rated better than those of L0 and L10. However, no differences were detected in color, flavor, and aroma attributes from sensory evaluation. Lipid oxidation was increased with increasing the contents of $n \bar{i} 3$ PUFA and postmortem aging time.
\end{abstract}

Keyword: Goat; n ī 3 fatty acids; Meat quality; Aging time 